\title{
Ellagic acid and Sennoside B inhibit osteosarcoma cell migration, invasion and growth by repressing the expression of c-Jun
}

\author{
WEI XU ${ }^{1 *}$, JINJIN XU $^{2 *}$, TING WANG $^{1 *}$, WEIBO LIU $^{1}$, HAIFENG WEI $^{1}$, \\ XINGHAI YANG ${ }^{1}$, WANGJUN YAN ${ }^{1}$, WANG ZHOU ${ }^{1}$ and JIANRU XIAO ${ }^{1}$ \\ ${ }^{1}$ Department of Orthopedic Oncology, Changzheng Hospital, Second Military Medical University, Shanghai 200003; \\ ${ }^{2}$ Shanghai Key Laboratory of Regulatory Biology, Shanghai Key Laboratory of Brain Functional Genomics \\ (Ministry of Education), Institute of Biomedical Sciences, East China Normal University, Shanghai 200241, P.R. China
}

Received April 5, 2016; Accepted December 6, 2017

DOI: $10.3892 / \mathrm{ol} .2018 .8712$

\begin{abstract}
Osteosarcoma is a mesenchymally derived, high-grade bone sarcoma that is the most frequently diagnosed primary malignant bone tumor. Today, chemoprevention is regarded as a promising and realistic approach in the prevention of human cancer. Previous studies have suggested ellagic acid (EA) and Sennoside B have potential in this regard. The aim of the present study was to elucidate the anti-osteosarcoma effects of EA and Sennoside B by using Saos-2 and MG63 osteosarcoma cells. It was identified that EA or Sennoside B treatment could inhibit the growth, migration and invasion of the cells, and induce $G_{1}$ cell cycle arrest by repressing the transcription of c-Jun. These results may provide a cellular basis for the application of EA or Sennoside B in the treatment of patients with osteosarcoma.
\end{abstract}

\section{Introduction}

Osteosarcoma (OS) is one of the most malignant types of bone tumor, which is most frequent in children and adolescents and characterized by the formation of neoplastic bone tissue (1). There is a high tendency to for OS to undergo metastasis (2). The treatment of OS typically includes surgery, radiation, chemotherapy, or the combination of chemotherapy and radiotherapy; however, these treatments are not successful in the long-term (3). Numerous studies

Correspondence to: Dr Jianru Xiao or Dr Wang Zhou, Department of Orthopedic Oncology, Changzheng Hospital, Second Military Medical University, 415 Fengyang Road, Shanghai 200003, P.R. China

E-mail: jianruxiao83@163.com

E-mail: brilliant212@163.com

${ }^{*}$ Contributed equally

Key words: osteosarcoma, ellagic acid, sennoside B, migration, growth, c-Jun have indicated the abilities of natural products to inhibit the development of different types of cancer in multiple animal models, as previously reviewed (4). These compounds may be used as anti-proliferation or anti-metastasis agents.

Sennoside, an extract from senna, is widely used as a stimulant laxative and its safety and efficiency have been established (5); however, little is known regarding its usefulness as an anti-tumor agent. Ellagic acid (EA) is a polyphenol compound with strong antioxidant properties that is found as ellagitannins in the fruits and nuts of several plants. The oral administration of EA can protect the system from alcohol toxicity by decreasing the expression of liver marker enzymes and increasing the activity of the antioxidant cascade $(6,7)$; it was previously demonstrated to induce apoptosis and cell cycle arrest in various types of cancer cell (8-11). However, its potential anti-tumor role in osteosarcoma and the mechanisms for its effects remain elusive.

The c-Jun N-terminal kinase (JNK) signal transduction pathway is associated with malignant cellular transformation (12). Studies with cultured cells have demonstrated that the JNK signal transduction pathway participates in the proliferation, differentiation, apoptosis and metastasis of osteoblasts (13). Phosphorylated JNKs activate the Jun proto-oncogene, AP-1 transcription factor subunit (c-Jun), which homodimerizes and/or heterodimerizes with Fos proto-oncogene, AP-1 transcription factor subunit to generate the activator protein-1 transcription complex (AP-1) (14), which binds to specific DNA sequences at target promoters, and regulates the expression of cognate genes that participate in the differentiation and function of osteoblasts, as well as in the pathogenesis of osteosarcoma (15-17).

It the present study, it is reported that EA and Sennoside B can inhibit Saos-2 and MG63, two malignant osteosarcoma cell lines, growth, migration and invasion, and induce cell cycle arrest by the inhibition of c-Jun expression.

\section{Materials and methods}

Antibodies and reagents. Rabbit anti-poly(ADP-ribose) polymerase (PARP; cat no. 88817), p-p38 (cat no. 4511), p-extracellular signal-regulated kinase (ERK) 1/2 (cat no. 4370) and c-Jun (cat no. 9165) antibodies were obtained 
from Cell Signaling Technology, Inc. (Danvers, MA, USA); mouse anti-GAPDH (cat no. sc-32233) was purchased from Santa Cruz Biotechnology, Inc. (Dallas, TX, USA). The EA, Sennoside B and propidium iodide were obtained from Sigma-Aldrich (Merck KGaA, Darmstadt, Germany).

Cell culture. Saos-2 and MG63 human osteosarcoma cells were obtained from the American Type Culture Collection (Manassas, VA, USA) and were maintained in Dulbecco's modified Eagle's medium (DMEM; Hyclone; GE Healthcare Life Sciences, Logan, UT, USA) with $10 \%$ fetal bovine serum (Hyclone; GE Healthcare Life Sciences), $100 \mathrm{U} / \mathrm{ml}$ penicillin and $100 \mu \mathrm{g} / \mathrm{ml}$ streptomycin at $37^{\circ} \mathrm{C}$ in a humidified $5 \% \mathrm{CO}_{2}$ atmosphere.

Wound closure assay. Saos-2 and MG63 cells were plated in a 12 -well cell culture plate. When the cell density reached $\geq 90 \%$, the monolayer was scratched with a $200 \mu \mathrm{l}$ pipette. Wound closure was monitored with phase contrast microscopy and quantified as the relative wound closure rate (wound distance at a specific time point/the original wound distance).

Transwell assay. A total of $1 \times 10^{4}$ of Saos- 2 or MG63 cells were seeded in the upper chamber of Transwell plates (BD Bioscience) with Matrigel, in serum-free conditions. DMEM supplemented with $10 \%$ fetal calf serum (Hyclone; GE Healthcare Life Sciences) and $50 \mathrm{ug} / \mathrm{ml}$ fibronectin (BD Biosciences, Franklin Lakes, NJ, USA) was used as a chemoattractant in the lower chamber. At $48 \mathrm{~h}$, cells remaining on the upper chamber were removed with a cotton swab, while cells adhering to the lower membrane were stained with $0.1 \%$ crystal violet for $30 \mathrm{~min}$ at room temperature and photographed with an inverted microscope (Zeiss AG, Oberkochen, Germany). The area of positive staining was measured using image analysis software (Image-Pro Plus 6.0; Media Cybernetics, Inc., Rockville, MD, USA); the rate of invasion was calculated as the positive area percentage. At least three independent experiments were performed for each condition.

MTT assays. Cell viability was assessed with an MTT assay in replicates. Cells were seeded in 96 -well plates at $2.5 \times 10^{3}$ cells per well (Saos-2 cells) or $1.0 \times 10^{3}$ cells per well (MG63 cells) and incubated for $0,24,48$ or $72 \mathrm{~h}$. Then the medium was replaced with $200 \mathrm{ml}$ fresh DMEM containing $0.5 \mathrm{mg} / \mathrm{ml}$ MTT, the cells were incubated for a further $4 \mathrm{~h}$, then MTT formazan crystals were dissolved in DMSO and absorbance at $490 \mathrm{~nm}$ was measured and analyzed.

Reverse transcription-quantitative polymerase chain reaction $(R T-q P C R)$. To assess mRNA levels, RNA was isolated from cells using TRIzol reagent (Takara Biotechnology Co., Ltd., Dalian, China) and cDNA was synthesized using the MLV reserve transcriptase from Promega Corporation (Madison, WI, USA), according to the manufacturers' protocols. The cDNA was subjected to qPCR $\left(95.0^{\circ} \mathrm{C}\right.$ for $10 \mathrm{~min}$ for 1 cycle, then $95.0^{\circ} \mathrm{C}$ for $15 \mathrm{sec}$ followed by $60.0^{\circ} \mathrm{C}$ for $1 \mathrm{~min}$ for 40 cycles) using SYBR-Green master mix (Toyobo Life Science, Osaka, Japan) in the Mx3005P Real-Time PCR system (Stratagene;
Agilent Technologies, Inc., Santa Clara, CA, USA). The comparative cycle quantification method $\left(2^{\Delta \mathrm{CC}}\right)$ was employed to analysis the gene expression with housekeeping gene $18 \mathrm{~S}$ as an internal normalization control (18). Each experiment was performed in duplicate and repeated three times. The primers for qPCR were as follows: c-Jun forwards, TCCAAG TGCCGAAAAAGGAAG; reverse, CGAGTTCTGAGCTTT CAAGGT; 18S forwards, GGACACGGACAGGATTGACA; reverse, GACATCTAAGGGCATCACAG. I8S was used as an internal control.

Cell cycle analysis. Saos- 2 cells or MG63 cells were treated with EA or Sennoside B for $24 \mathrm{~h}$. Cells were trypsinized, collected, fixed and stained with propidium iodide solution following the manufacturer's protocol of the Propidium Iodide Flow Cytometry kit (cat no. ab139418, Abcam, Cambridge, UK). The cell cycle distribution was then analyzed by the BD FACSCalibur (BD Biosciences) using CellQuest Pro, version 5.1 (BD Biosciences).

Western blotting. Saos-2 or MG63 cells were treated with EA or Sennoside B for $24 \mathrm{~h}$, then scraped into ice-cold PBS and lysed with lysis buffer containing $50 \mathrm{mM}$ Tris- $\mathrm{HCl}$ ( $\mathrm{pH} 7.5), 1 \mathrm{mM}$ EDTA, $1 \%$ Nonidet P-40, $150 \mathrm{mM} \mathrm{NaCl}, 10 \%$ glycerol and protease inhibitors. The protein concentration was determined using the Pierce BCA protein assay kit (cat no. 23225, Thermo Fisher Scientific, Inc., Waltham, MA, USA). Same quantities of protein (25 $\mu \mathrm{g} / \mathrm{lane})$ were resolved using 12\% PAGE-SDS gel, transferred to a nitrocellulose membrane (Bio-Rad Laboratories, Inc., Hercules, CA, USA; cat no. 1620112), then followed by $5 \%$ non-fat milk blocking at room temperature for $1 \mathrm{~h}$. The proteins were analyzed with antibodies against PARP (1:1,000 diluted in 5\% BSA), p-ERK (1:2,000 diluted in 5\% BSA), c-Jun (1:1,000 diluted in 5\% BSA), p-p38 (1:1,000 diluted in 5\% BSA), and GAPDH (1:1,000 diluted in 5\% BSA). The primary antibodies were incubated with membrane at $4^{\circ} \mathrm{C}$ overnight, followed by a $1 \mathrm{~h}$ secondary antibody incubation at room temperature. HRP-conjugated goat anti-mouse (Jackson ImmunoResearch Laboratories, Inc., West Grove, PA, USA; cat no. 115-035-146; 1:5,000 in 5\% non-fat milk) and goat anti-rabbit (Jackson ImmunoResearch Laboratories, Inc.; cat no. $115-035-045 ; 1: 5,000$ in 5\% non-fat milk) secondary antibodies were used to visualize the protein of interest using Amersham ECL Prime Western Blotting Detection Reagent (cat no. RPN2236; GE Healthcare, Chicago, IL, USA). All data were the results from 3 replicates.

Statistics. Statistical analysis was performed with GraphPad Prism 6.0 (GraphPad Software, Inc., La Jolla, CA, USA). Quantitative data are expressed as the means \pm standard deviation. Statistical analysis was performed with a one-way analysis of variance followed by Dunnett's multiple comparisons test. $\mathrm{P}<0.05$ was considered to indicate a statistically significant difference.

\section{Results}

EA and Sennoside B inhibit the migration and invasion of osteosarcoma cells. Previous reports have indicated the anti-tumor potential of EA and Sennoside B (8). In order to 
A
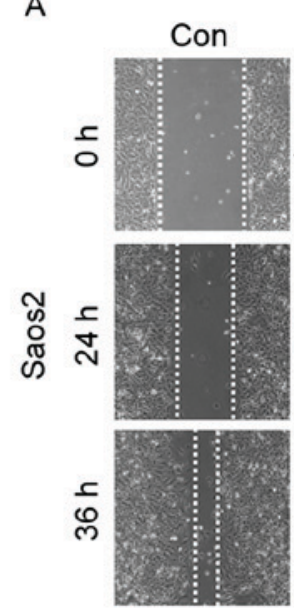

C

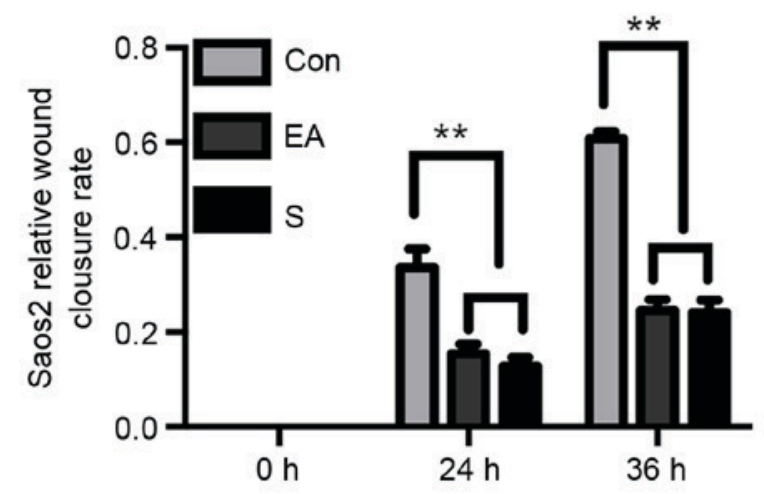

E
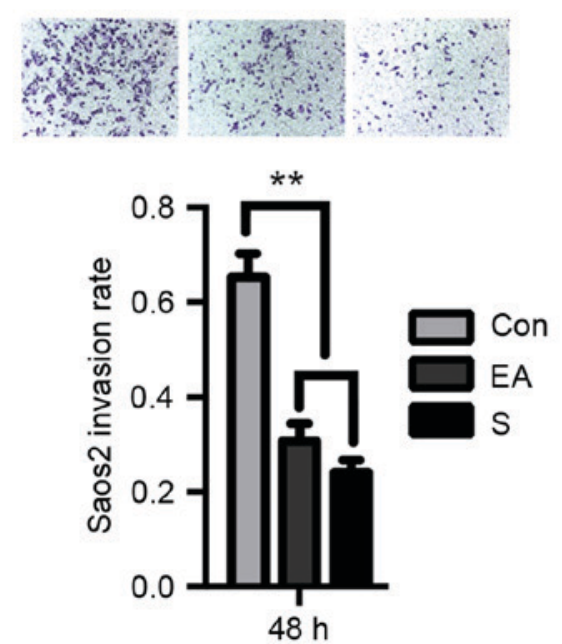

B
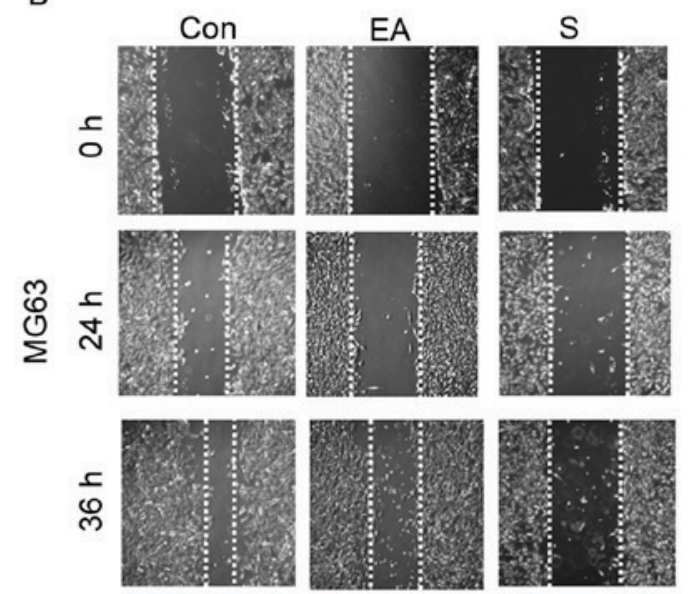

D

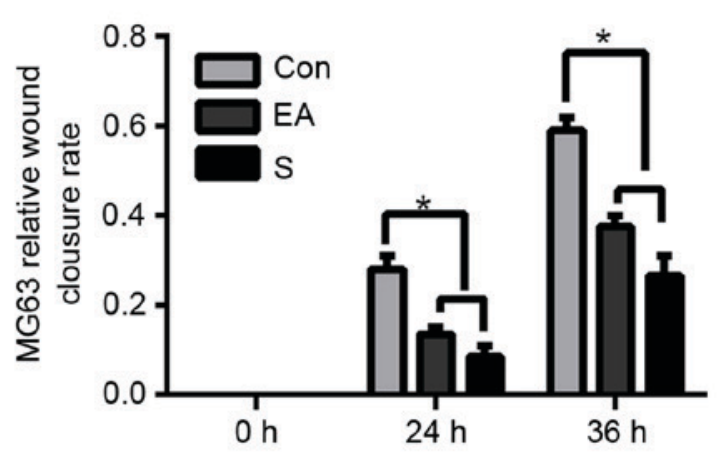

F
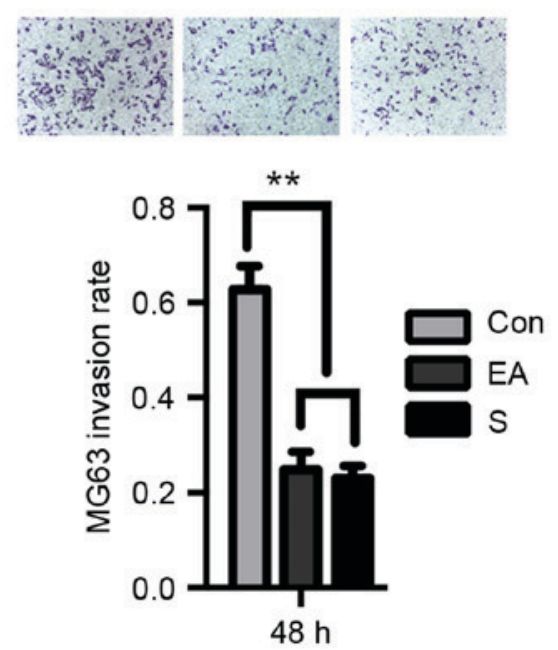

Figure 1. EA and Sennoside B inhibit the migration of osteosarcoma cells. (A) Saos-2 or (B) MG63 cells were treated with $20 \mu \mathrm{M}$ EA or Sennoside B, and wound closure was monitored. Magnification, x10. The closure rate was quantified for (C) Saos-2 and (D) MG63 cells in order to compare the rate of migration. (E) Saos-2 or (F) MG63 cells were seeded on transwell membranes with or without EA or Sennoside B treatment. Magnification, x20. The migrated cells were fixed and stained with crystal violet (upper panel). The proportion of migrated cells was then quantified (lower panel). Data represent the mean \pm standard deviation of 3 experimental repeats. ${ }^{*} \mathrm{P}<0.05,{ }^{* *} \mathrm{P}<0.01$. EA, ellagic acid; Con, control; S, Sennoside B.

investigate their effect on osteosarcoma, Saos-2 and MG63 cells were analyzed. A series of concentrations of each agent were used in a wound-healing assay to verify their influence on the migration of OS cells. It was indicated that the $20 \mu \mathrm{M}$ EA or Sennoside B was the minimal requirement for the inhibition of the migration ability of Saos-2 cells (data not shown and Fig. 1A), which was consistent with the result for MG63 cells (data not shown and Fig. 1B). The analysis of the wound-healing assay demonstrated that when the cells were exposed to $20 \mu \mathrm{M}$ EA or Sennoside B for 24 or $36 \mathrm{~h}$, the inhibition rate was $40-70 \%$ (Fig. $1 \mathrm{C}$ and $\mathrm{D}$; Saos-2, $\mathrm{P}<0.01$; MG63, $\mathrm{P}<0.05)$. Then, the invasion abilities of these cells in 
A

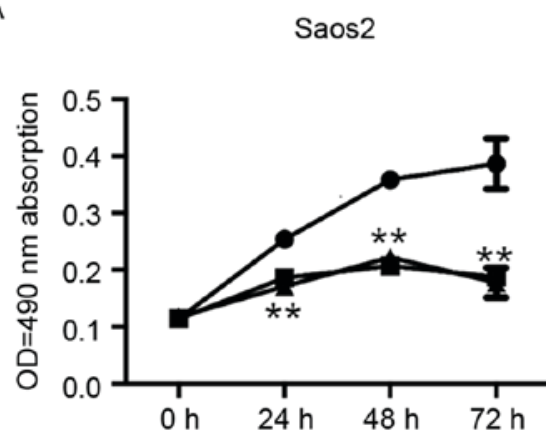

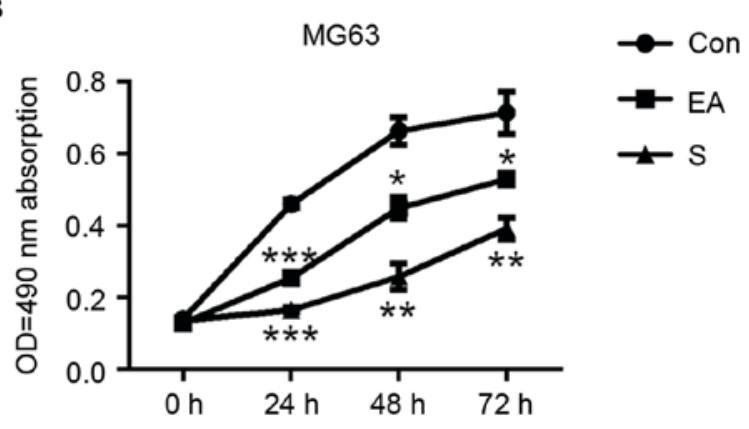

D

C

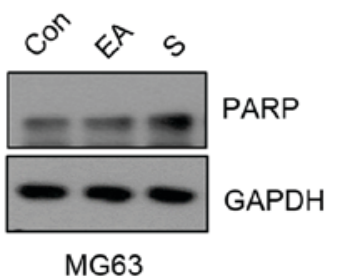

Figure 2. EA and Sennoside B inhibit osteosarcoma cell growth. (A) Saos-2 or (B) MG63 cells were cultured with $20 \mu$ M EA or Sennoside B for the indicated times. The rate of cell growth was determined by MTT assays. Data represent the mean \pm standard deviation of 3 experimental repeats performed in triplicate. ${ }^{* *} \mathrm{P}<0.01,{ }^{* * *} \mathrm{P}<0.0001$. The (C) Saos-2 or (D) MG63 cells were treated with $20 \mu \mathrm{M}$ EA or Sennoside B for $24 \mathrm{~h}$. The indicated proteins were detected by western blotting; representative images are included. EA, ellagic acid; Con, control; S, Sennoside B.

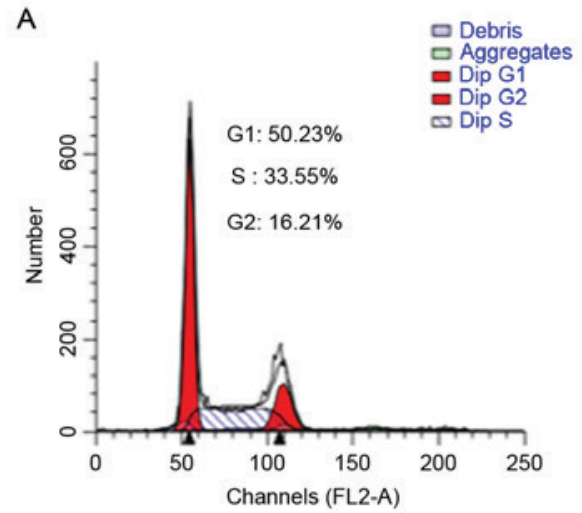

Con

B

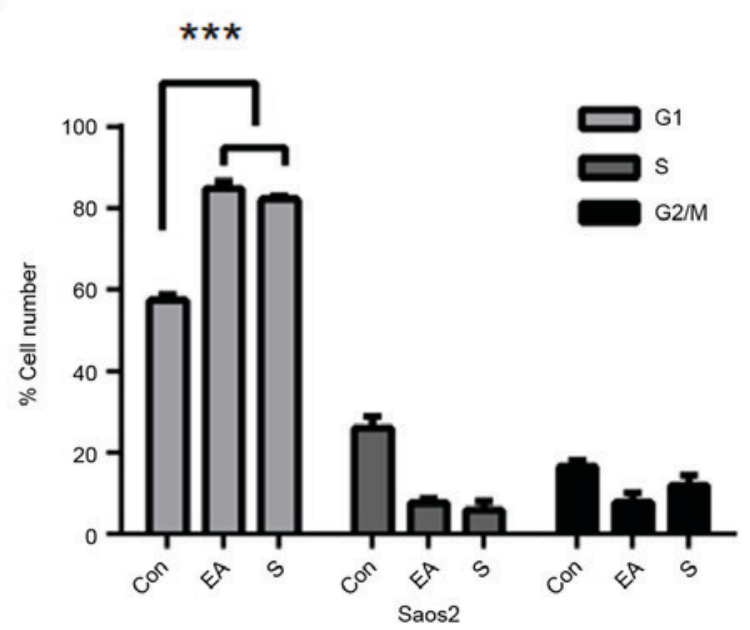

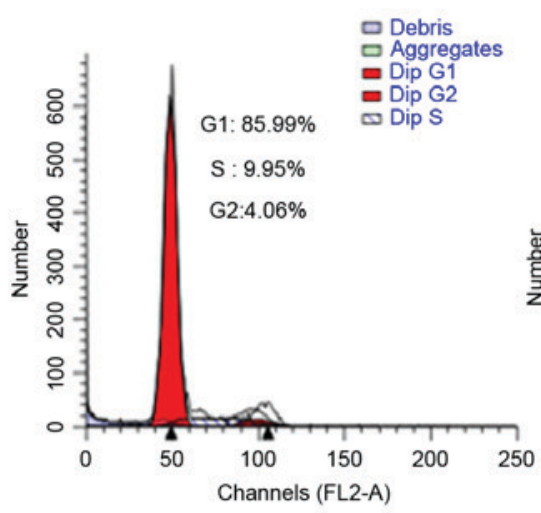

EA

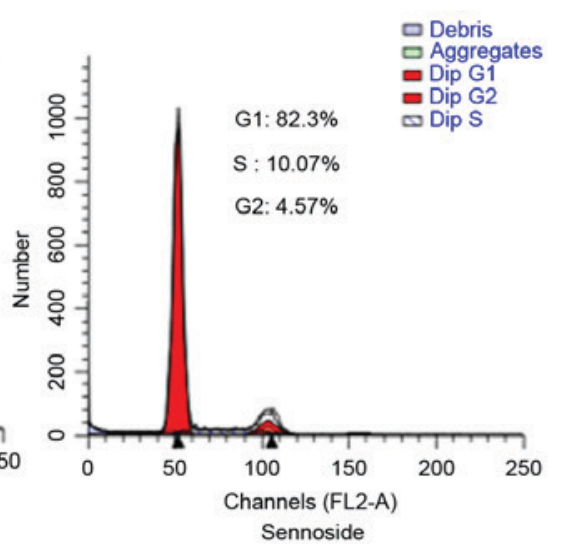

Sennoside

C

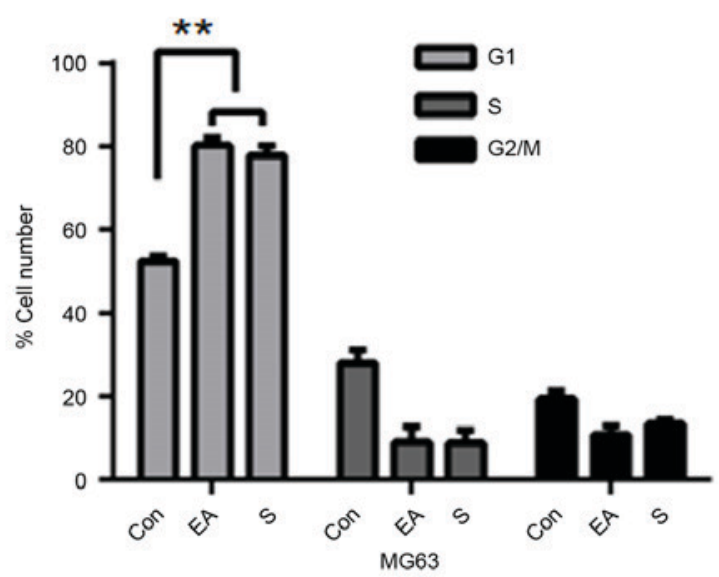

Figure 3. EA and Sennoside B induce cell cycle arrest in osteosarcoma cells. (A) Cells were treated with EA or Sennoside B for $24 \mathrm{~h}$, stained with propidium iodide and analyzed by fluorescence-activated cell sorting. A representative image with Saos-2 cells is depicted. The compounds induced the accumulation of (B) Saos-2 and (C) MG63 cells in the $\mathrm{G}_{1}$ phase. Data represent the mean \pm standard deviation of 3 experimental repeats. ${ }^{* * *} \mathrm{P}<0.01,{ }^{* * * *} \mathrm{P}<0.0001$. EA, ellagic acid; Con, control; S, Sennoside B. 
A

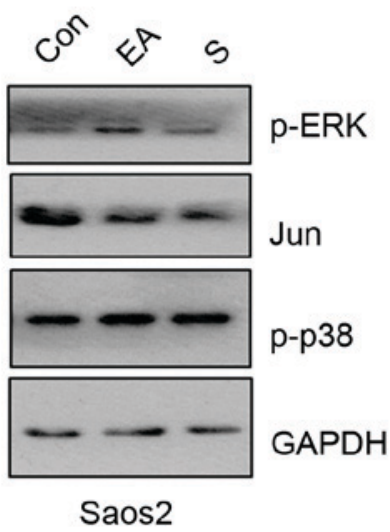

C

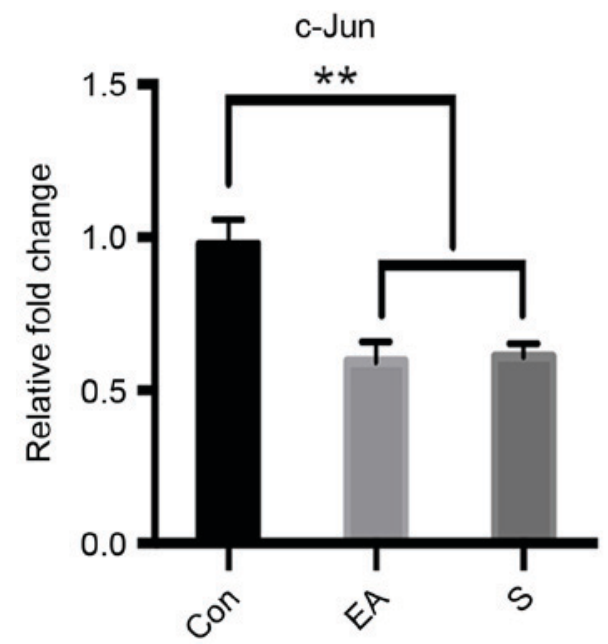

B

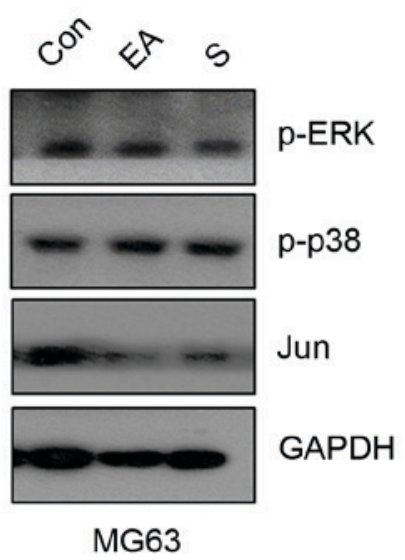

D

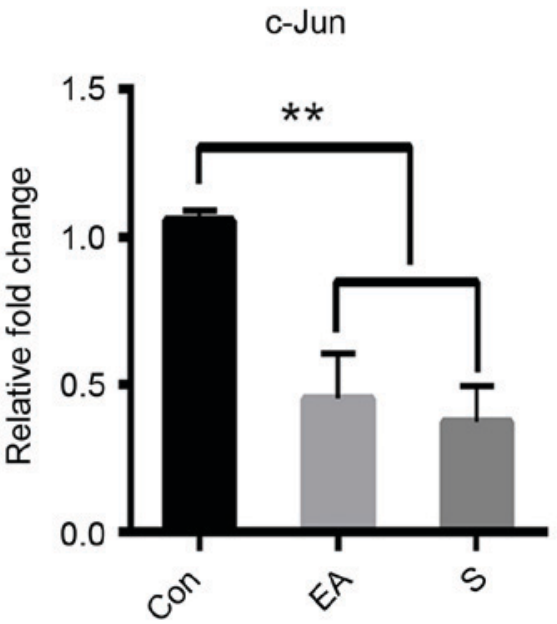

Figure 4. EA and Sennoside B inhibit c-Jun expression in osteosarcoma cells. (A and B) Following $24 \mathrm{~h}$ treatment with EA or Sennoside B, the indicated proteins were detected by western blotting in (A) Saos-2 and (B) MG63 cells. The (C) Saos-2 or (D) MG63 cells were treated with EA or Sennoside B for $12 \mathrm{~h}$ before the total RNA was extracted and the c-Jun mRNA expression level was examined by reverse transcription-quantitative polymerase chain reaction with specific primers. Data represent the mean \pm standard deviation of 3 experimental repeats performed in duplicate. ** P<0.01. EA, ellagic acid; c-Jun, Jun proto-oncogene, AP-1 transcription factor subunit; Con, control; S, Sennoside B; p-, phosphorylated-; ERK, extracellular signal-regulated kinase.

the presence or absence of EA or Sennoside B were examined by transwell assays. The EA- or Sennoside B-treated cells invaded significantly less frequently than the control cells (Fig. 1E and F; $\mathrm{P}<0.01$ ). Thus, it was determined that EA or Sennoside B treatment can suppress the migration and invasion of osteosarcoma cells.

$E A$ and Sennoside B inhibit the growth of osteosarcoma cells without inducing apoptosis. Saos-2 and MG63 cells were treated with $20 \mu \mathrm{M}$ EA or Sennoside B, and viability was examined by an MTT assay. The results suggested both EA and Sennoside B significantly inhibited cell growth from $24 \mathrm{~h}$ at $20 \mu \mathrm{M}$, compared with the control groups (Fig. 2A and B; Saos-2, $\mathrm{P}<0.01$; MG63, $\mathrm{P}<0.0001)$. To assess whether the induction of apoptosis served a role in the repression of the proliferation of OS cells, the cells were subjected to EA and Sennoside B treatment for $24 \mathrm{~h}$ and were collected for western blotting. No significant difference in cleaved PARP expression was observed between the control and treated groups (Fig. 2C and D), even though a previous study demonstrated that EA inhibited proliferation by the induction of apoptosis in colon and prostate cancer cells (19).

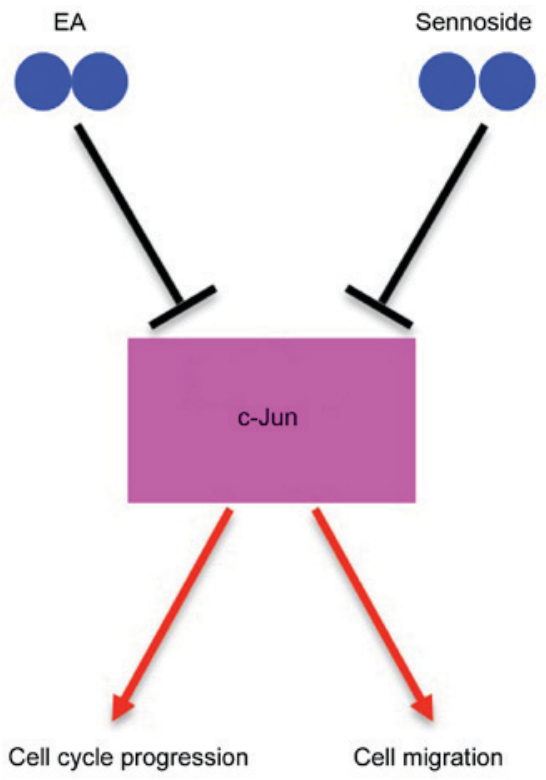

Figure 5. A schematic of the potential mechanism for the inhibition of the migration and growth of osteosarcoma cells by EA or Sennoside B. EA, ellagic acid; c-Jun, Jun proto-oncogene, AP-1 transcription factor subunit. 
EA and Sennoside B induce cell cycle arrest. As EA and Sennoside B inhibited cell growth without inducing apoptosis, the cell cycle distribution of cells treated with $20 \mu \mathrm{M}$ EA or Sennoside B for $24 \mathrm{~h}$ was analyzed. It was identified that $>80 \%$ of Saos- 2 cells in the treated groups were in the $\mathrm{G}_{1}$ stage, compared with $<60 \%$ in the control groups (Fig. 3A and B; $\mathrm{P}<0.0001)$. Similar results were detected for MG63 cells (Fig. 3C; $\mathrm{P}<0.01$ ).

EA and Sennoside B repress c-Jun expression. The accumulation of cells in the $G_{1}$ phase indicated the inhibition of the signals required for cell cycle progression. The expression of factors from key signaling pathways associated with $\mathrm{G}_{1}$ cell cycle progression was thus examined following exposure to EA or Sennoside B. After $24 \mathrm{~h}$ of treatment, the c-Jun protein level was observed to decrease to a greater extent than the other assessed proteins (Fig. 4A and B). In order to further confirm the effect of EA or Sennoside B on c-Jun, mRNA was extracted for RT-qPCR following the same treatment. These results suggested that EA or Sennoside B treatment repressed the transcription of c-Jun mRNA (Fig. 4C and D). A proposed schematic was drawn to represent the potential mechanism for the EA or Sennoside B inhibition of the growth, migration and invasion of osteosarcoma cells (Fig. 5).

\section{Discussion}

In the present study, it was demonstrated that both EA and Sennoside B treatment can inhibit cell migration, invasion and proliferation. The inhibitory effect of EA or Sennoside B on proliferation was attributed to the induction of cell cycle arrest. The data regarding the effects of EA on osteosarcoma are consistent with previous studies in other cancer types (7-11). However, the inhibitory effects of Sennoside B were demonstrated for the first time, to the best of our knowledge.

The high incidence of mortality in osteosarcoma is associated with tumor metastasis (1). Metastasis is a complex cascade, including various physiological alterations to induce extracellular matrix (ECM) degradation (20). Matrix metallopeptidases (MMPs), particularly MMP-2 and MMP-9, have been reported as the most important factors in the degradation of type IV collagen, a major component of the basement membrane, which induces tumor cell growth and metastasis (21). It has been reported that EA inhibits either MMP2 activity or the secretion of collagenases and gelatinases (22). AP-1 may serve a dominant role in the transcriptional activation of the MMP promoters, and increasing evidence has indicated the key role of c-Jun in the regulation of MMPs (23). In the present study, both EA and Sennoside B treatment repressed the transcription of c-Jun, ultimately leading to a decrease in its protein level. This mechanism partially explains why two different compounds inhibited Saos-2 and MG63 cells in a similar manner.

Previous studies indicated that EA could inhibit cancer cell growth by inducing apoptosis and cell cycle arrest $(8-11,19)$, but Sennoside B's effect on cell growth was previously unreported. The results of the present study suggested that both EA and Sennoside B could inhibit cell proliferation without inducting apoptosis. The oncogenic protein c-Jun regulates a range of cell cycle progression-associated genes, including cyclin D1 (24). Cell cycle arrest in the present study was likely to be caused by the repression of c-Jun expression. However, other factors can induce the cell cycle arrest of osteosarcoma cells. For example, the knockdown of ROR2 in osteosarcoma cells inhibited cell proliferation and colony formation by inducing cell cycle arrest (25). In addition, it was previously reported that the exogenous expression of miR-497 in human osteosarcoma MG63 cells suppressed cell proliferation, colony formation, migration and invasion, and induced apoptosis and arrest at the $G_{0} / G_{1}$ phase of the cell cycle (26).

In conclusion, it was identified that EA and Sennoside B may share a common mechanism to inhibit the growth, migration and invasion of Saos-2 and MG63 cells, specifically, the repression of c-Jun expression at the mRNA level. Further studies are required to understand the detailed molecular mechanisms of action of EA and Sennoside B on the regulation of the expression of c-Jun, which may provide useful information for their possible application in osteosarcoma prevention and therapy.

\section{Acknowledgements}

The present study was supported by the Shanghai Youth Science and Technology Talent Sailing Program (grant no. 14YF1405900).

\section{References}

1. Wermers RA, Tiegs RD, Atkinson EJ, Achenbach SJ and Melton LJ III: Morbidity and mortality associated with Paget's disease of bone: A population-based study. J Bone Miner Res 23: 819-825, 2008

2. Mirabello L, Troisi RJ and Savage SA: Osteosarcoma incidence and survival rates from 1973 to 2004: Data from the surveillance, epidemiology and end results program. Cancer 115: 1531-1543, 2009.

3. Liao CL, Lai KC, Huang AC, Yang JS, Lin JJ, Wu SH, Gibson Wood W, Lin JG and Chung JG: Gallic acid inhibits migration and invasion in human osteosarcoma U-2 OS cells through suppressing the matrix metalloproteinase-2/-9, protein kinase $\mathrm{B}$ (PKB) and PKC signaling pathways. Food Chem Toxicol 50: 1734-1740, 2012

4. Hong WK and Sporn MB: Recent advances in chemoprevention of cancer. Science 278: 1073-1077, 1997.

5. Ishibashi K, Kumamoto K, Kuwabara K, Hokama N, Ishiguro T, Ohsawa T, Okada N, Miyazaki T, Yokoyama M, Tsuji Y, et al: Usefulness of sennoside as an agent for mechanical bowel preparation prior to elective colon cancer surgery. Asian J Surg 35: 81-87, 2012.

6. Hussein RH and Khalifa FK: The protective role of ellagitannins flavonoids pretreatment against $\mathrm{N}$-nitrosodiethylamine induced-hepatocellular carcinoma. Saudi J Biol Sci 21: 589-596, 2014.

7. Sohn EH, Koo HJ, Hang DTT, Jang SA, Namkoong S, Lim JD and Kang SC: Protective effects of ellagic acid on ethanol-induced toxicity in hepatic HepG2 cells. Mol Cell Toxicol 9: 249-256, 2013.

8. Li TM, Chen GW, Su CC, Lin JG, Yeh CC, Cheng KC and Chung JG: Ellagic acid induced p53/p21 expression, G1 arrest and apoptosis in human bladder cancer T24 cells. Anticancer Res 25: 971-979, 2005.

9. Mertens-Talcott SU and Percival SS: Ellagic acid and quercetin interact synergistically with resveratrol in the induction of apoptosis and cause transient cell cycle arrest in human leukemia cells. Cancer Lett 218: 141-151, 2005.

10. Narayanan BA, Geoffroy O, Willingham MC, Re GG and Nixon DW: p53/p21(WAF1/CIP1) expression and its possible role in G1 arrest and apoptosis in ellagic acid treated cancer cells. Cancer Lett 136: 215-221, 1999. 
11. Päivärinta E, Pajari AM, Törrönen R and Mutanen M: Ellagic acid and natural sources of ellagitannins as possible chemopreventive agents against intestinal tumorigenesis in the Min mouse. Nutr Cancer 54: 79-83, 2006

12. Whitmarsh AJ and Davis RJ: Transcription factor AP-1 regulation by mitogen-activated protein kinase signal transduction pathways. J Mol Med (Berl) 74: 589-607, 1996.

13. Papachristou DJ, Batistatou A, Sykiotis GP, Varakis I and Papavassiliou AG: Activation of the JNK-AP-1 signal transduction pathway is associated with pathogenesis and progression of human osteosarcomas. Bone 32: 364-371, 2003.

14. Angel P and Karin M: The role of Jun, Fos and the AP-1 complex in cell-proliferation and transformation. Biochim Biophys Acta 1072: 129-157, 1991

15. Hipskind RA and Bilbe G: MAP kinase signaling cascades and gene expression in osteoblasts. Front Biosci 3: d804-d816, 1998.

16. Liu B and Shuai K: Induction of apoptosis by protein inhibitor of activated Stat1 through c-Jun NH2-terminal kinase activation. J Biol Chem 276: 36624-36631, 2001.

17. Suzuki A, Guicheux J, Palmer G, Miura Y, Oiso Y, Bonjour JP and Caverzasio J: Evidence for a role of p38 MAP kinase in expression of alkaline phosphatase during osteoblastic cell differentiation. Bone 30: 91-98, 2002.

18. Livak KJ and Schmittgen TD: Analysis of relative gene expression data using real-time quantitative PCR and the 2(-Delta Delta C(T)) method. Methods 25: 402-408, 2001.

19. Umesalma S, Nagendraprabhu P and Sudhandiran G: Ellagic acid inhibits proliferation and induced apoptosis via the Akt signaling pathway in HCT-15 colon adenocarcinoma cells. Mol Cell Biochem 399: 303-313, 2015.
20. Hanahan D and Weinberg RA: The hallmarks of cancer. Cell 100: 57-70, 2000.

21. Chambers AF and Matrisian LM: Changing views of the role of matrix metalloproteinases in metastasis. J Natl Cancer Inst 89: 1260-1270, 1997.

22. Pitchakarn P, Chewonarin T, Ogawa K, Suzuki S, Asamoto M, Takahashi S, Shirai T and Limtrakul P: Ellagic acid inhibits migration and invasion by prostate cancer cell lines. Asian Pac J Cancer Prev 14: 2859-2863, 2013.

23. Chakraborti S, Mandal M, Das S, Mandal A and Chakraborti T: Regulation of matrix metalloproteinases: An overview. Mol Cell Biochem 253: 269-285, 2003.

24. Bakiri L, Lallemand D, Bossy-Wetzel E and Yaniv M: Cell cycle-dependent variations in c-Jun and JunB phosphorylation: A role in the control of cyclin D1 expression. EMBO J 19: 2056-2068, 2000.

25. Huang J, Shi Y, Li H, Tan D, Yang M and Wu X: Knockdown of receptor tyrosine kinase-like orphan receptor 2 inhibits cell proliferation and colony formation in osteosarcoma cells by inducing arrest in cell cycle progression. Oncol Lett 10: 3705-3711, 2015.

26. Ge L, Zheng B, Li M, Niu L and Li Z: MicroRNA-497 suppresses osteosarcoma tumor growth in vitro and in vivo. Oncol Lett 11: 2207-2212, 2016.

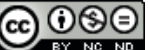

This work is licensed under a Creative Commons

Attribution-NonCommercial-NoDerivatives 4.0 International (CC BY-NC-ND 4.0) License. 